\title{
Tips for GP trainees working in paediatrics
}

One of the most useful rotations that a GP registrar can undertake is a stint in paediatrics. This is particularly true if you do not have your own children, or much experience interacting with children. It is a rare day in general practice that doesn't involve some paediatrics so its good to gain experience in an environment where there is senior help at hand, as well as experienced nursing staff to offer advice and support. It is fair to say that many registrars start this rotation with some sense of foreboding and fear, worried that they will have to take responsibility for the health of acutely sick children without help. It is the author's experience that this is not the norm. Paediatric departments do not expect their new SHOs to have much knowledge of the area. They do, though, expect you to assess children thoroughly, safely and sensitively, ideally without getting the parents offside, and then to discuss patients with a senior if you have any questions or doubts. It's probably sensible to discuss all patients you see, at least initially. Access to consultants is usually good compared to other specialties, so make the most of this learning environment while you are there.

The following list, while not exhaustive, may help to allay some of the fears of the GP registrar as they approach the job. The list is unapologetically based on the author's experience of the paediatric rotation rather than guidelines or policy.

1. Before the start of the placement revise the management of common presentations in children (abdominal pain, urinary tract infection, constipation, rash, pyrexia, croup, chest infection, asthma, and seizures).

2. When clerking in a child always ask about family history, social history, and immunisations.

3. Ask for a prompt senior review if you have any concerns regarding a child's safety at home and school. If you have concerns don't ignore them, talk to a senior.
4. Cannulating a child can be terrifying for all concerned; the child, the parent and the SHO! As in all things, experience improves performance. In time you will pick up this skill. In the meantime, your registrar will probably expect you to have had a go before calling on them for help.

5. Always have all equipment ready and distract a child with a helpful member of staff before inserting a cannula/taking blood from a child.

6. Never tell a child you are going to insert a needle - refer to it as a blood test or spaghetti (when cannulating young children).

7. When prescribing check and recheck all doses in the BNF for Children.

8. Become confident and competent in delivering intramuscular injections to babies and children during the placement as this is a useful skill to have in general practice.

9. When taking GP referrals always ask the referring GP how sick the child is and to send very sick babies/children to A\&E for you to assess there. All blue-light referrals should be seen in a resus environment rather than on the ward.

10. Don't be rude to GPs referring in from a primary care setting devoid of paediatric nurses, paediatric sats probes, and piped oxygen. Bear in mind that you may have more acute paediatric experience than they do and that they do not have the luxury to observe a child for 2 hours taking regular obs.

11. If covering neonates, ask the registrar to attend the first few 'at risk' deliveries with you until you feel confident enough to attend by yourself.

12. Try not to panic when you are called to a difficult delivery, neonatal resuscitation is generally easier than adult.

13. When waiting for a Caesarean Section to take place on a preterm baby, use the time to start gathering the required maternal and paternal information for admission to the neonatal unit.
14. Attend a NLS (Newborn Life Support) course as soon as you can after starting (or even better, before you start).

15. It's probably best to have your first few new baby checks supervised by a senior until you develop a system of examination that you are confident with. Take this opportunity to get it signed off as a DOPS (Direct Observation of Procedural Skills).

16. When doing baby checks explain to parents that it is a screening test and be prepared to change a nappy as part of it!

17. During the baby check remember that, no matter how tired or stressed you are, this is a big day for the child and a big day for mum (who has gone through a lot). Don't be the grumpy paediatric $\mathrm{SHO}$ who spoils it. Be professional and thorough but try to also be bright and positive (even if this is an act).

18. Before you start make sure you know what hypospadias and talipes look like (there are lots of pictures on the internet).

19. Learn the local pathway for referral of hypospadias, talipes, and clicking hips.

20. Become familiar with the trust protocols for babies with hypoglycaemia, hypothermia, intrauterine growth restriction, and maternal diabetes.

21. When admitting a baby from the postnatal ward to the neonatal unit be open and honest with the parents and address any concerns they have at this time. Also make them aware of the visiting times and how and when to raise concerns. Spend your time on the neonatal ward improving your communication skills by talking to the parents. They often feel bewildered and scared and are often ignored by the doctors who are concentrating on the child.

22. Try not to be afraid of the kids. They and their parents will sense this. Try to be confident without being cocky.

23. Listen to the parents. Listen to the child. 
24. When you first see a patient, before you do anything else ask yourself if this looks like a sick child.

25. Don't be too quick to dismiss parental concern for parental over-anxiety.

26. Most children love stickers.

27. Don't rush in to examine a child in the non-emergency setting, spend a minute chatting to the parents. This will put the parents at ease, which will in turn put the child at ease. It also gives you the opportunity to observe the child which can be invaluable.

28. Don't discharge a febrile child on antibiotics without finding a source of infection.

29. If a parent says their child has been hot and unwell, then they have been regardless of their observations or presentation now (this isn't just good practice, it's NICE guidance').

30. Missing testicular torsion can be a disaster. It's always worth examining the genitals, with parental consent.

31. Check for nappy rash.

32. Examining the throat in children can be difficult. The Oxford Handbook of Emergency Medicine suggests using 'Dr Stick', a tongue depressor with a face drawn on it. It's amazing how many children will readily let $\mathrm{Dr}$ Stick examine their throat, especially if they think they will get to keep him afterwards (sleight of hand may be required to substitute a fresh depressor depending on the throat). ${ }^{2}$

33. Always do a full examination of children, including ear, nose, and throat; CRT (capillary refill time); heart rate; sats; and respiratory rate.

34. Learn from the paediatric nurses. If they say a child doesn't look right take this seriously and see the child quickly. Don't argue about the observations.

35. If you are doing paediatrics over winter be prepared for bronchiolitis. You will be very busy.

36. If doing paediatrics on Christmas Eve, ensure that, (where culturally appropriate), all admitted children are aware that you have passed on their location and details to Father Christmas and that Rudolph has no problems landing on the roof of the department. This small act can make a very big difference to both child and parent.

37. Meningococcal sepsis kills quickly. Be prepared to spot it and act quickly, involving senior help. Be suspicious of listless children with abnormal observations.

38. When moving a child into a high dependency or resus area in the acute setting keep the parents informed of where you are taking their child. This shouldn't get in the way of your medical attention to the patient.

39. Remembering normal values for paediatric vital signs can be difficult in stressful situations. It's often easier to laminate a ready reckoner that fits in your pocket. Some trusts provide this at induction. If yours doesn't, make your own.

40. Try to sit in on outpatient clinics, you will see the cases that in the future will be sitting in your consulting room.

\section{Matthew Burkes and \\ Alexander Goodman}

\section{REFERENCES}

1. National Institute for Health and Clinical Excellence. Feverish illness in children (CG47). Assessment and initial management in children younger than 5 years. http://egap.evidence.nhs.uk/CG47/ (accessed 23 Nov 2010).

2. Wyatt JP, Illingworth RN, Graham CA, et al. Oxford Handbook of Emergency Medicine. 3rd edn. Oxford: OUP, 2006.

DOI: 10.3399/bjgp11X549108 